\title{
Network Modeling and Analysis of Reconnaissance Satellite Application Information Chain System
}

\author{
Kangming Zhang ${ }^{1, a}$, Haoguang Chen ${ }^{1, b}$,Xueren Lin ${ }^{1, ~ c}$, Hao Zhang ${ }^{1, d}$ \\ ${ }^{1}$ Department of Graduate Management,Equipment Academy ,HuaiRou, Beijing, 101407,China \\ aemail: zkmnc@163.com, bchenhhaog@163.com,,530035717@qq.com, ${ }^{\mathrm{d}} 41198317 @ q q . c o m$,
}

Keywords: Reconnaissance Satellite Information Application Chain; Complex Network; Sub-graph; Super-edge; Loop

\begin{abstract}
Aiming at the problem of network model and analysis of reconnaissance satellite application information system, a modeling method is proposed based on complex network theory and a network analysis method is proposed based on task submap. This paper first defines the composition of reconnaissance satellite application information chain and constructs its task structure model. Secondly, it constructs the network model and puts forward the network analysis method of task subgraph. Finally, a simple model is used to verify the validity and applicability of the above methods.
\end{abstract}

\section{Introduction}

Reconnaissance satellite information application chain is the network structure which provides the reconnaissance surveillance information to the combat users in the course of war. It plays an important role in the target reconnaissance and strike assessment [1]. At present, the research on reconnaissance satellites in combat is mainly at the stratagem of strategy and campaign, and less on tactical level. The research on reconnaissance satellite system and the application of combat system is more, and less on the whole application process [2,3].The purpose of this paper is to consider the applications of reconnaissance satellite information from the perspective of the whole process and multiple levels, and to promote the tactical application transformation of reconnaissance satellite. It will promote the overall optimization of the reconnaissance satellites information application chain [4].

Based on the information chain composition structure and task structure model, this paper proposes a network modeling method and a network analysis method. And the model of the reconnaissance satellites application information chain is constructed by using the network theory and method [5]. Based on the information flow model and the combat ring[6], a network subgraph analysis method is proposed. These results have high value and significance for understanding the structure of reconnaissance satellite information application chain, studying its application flow and exploring its application.

\section{Structural Model of Reconnaissance Satellite Information Application Chain}

Reconnaissance satellite application information chain is a complex system, which consists of user systems and reconnaissance satellite systems. Each subsystem can independently perform certain task activities, but in the process of completing the system tasks, the subsystems must be closely coordinated. According to combat missions supported by reconnaissance satellites, the combat tasks of reconnaissance satellites information application chain contain four task types as follows: the surveillance task, the reconnaissance task, the evaluation task, and the situation task. In general, the completion of these tasks are completed by the integrated command and control activities, resource scheduling activities, information access activities, information relay activities, information receiving activities, information processing activities and information application 
activities. And these activities are supported by the reconnaissance satellite information application chain, whose performance level is determined by its structure and the capabilities of its nodes, which ultimately affect the combat mission, as shown in Figure. 1.

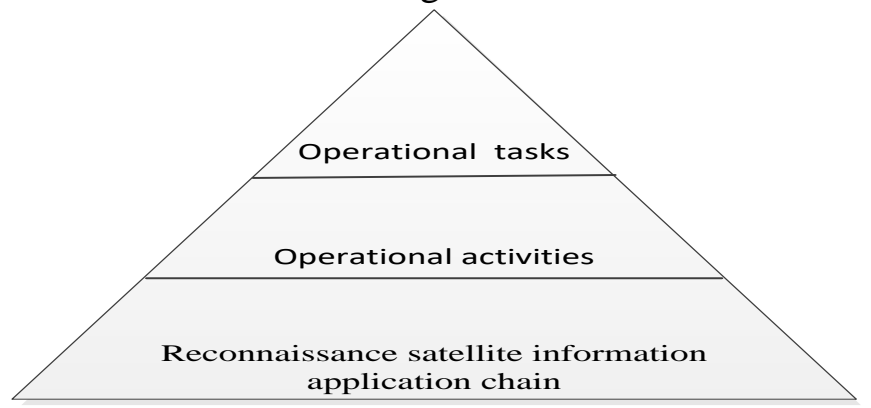

Fig. 1. Structural model of reconnaissance satellite information application chain

\section{Network Model of Reconnaissance Satellite Information Application Chain}

If the elements of the system are treated as nodes of the network, the relationship between the elements is seen as an edge, then a mapping relationship is established between the system and the network. Therefore, through the study of the corresponding network model of the system, the research purpose of the system can be achieved.

a) Network node description of reconnaissance satellite application information chain system

The information chain system network contains a variety of heterogeneous nodes. According to the function of nodes in the task activities, these nodes can be divided into the following 10 categories of nodes.

The information acquisition node (Ia) is composed of reconnaissance satellites. It is mainly responsible for the reconnaissance and surveillance of the target, the location and status of the target, and the information source for the combat users.

The information relay node (Ir)consists of some relay stars. Its function is to receive the target information from the reconnaissance satellite and transmit it to the ground receiving station.

The information receiving node consists of some ground receiving station nodes (Gr)and some relay star ground station nodes (Rg). Its function is to receive the target information from the reconnaissance satellite and transmit the information to the ground receiving station.

The information processing node(Ip) is mainly composed of some information processing system units. Its function is to processe the target information and form the information product.

The information distribution node (Id) is composed of distribution system units. The main function is to quickly and reliably distribute the target information to the combat users.

The combat user node $(\mathrm{Cu})$ is mainly composed of combat commander and combat weapon, which is mainly responsible for the demand of information and the application of information.

The integration task center node(Tc) is composed of the command management system unit. The main function is to analyze the information demand comprehensively, formulate the task planning, and execute the command.

The ground control center node (Mc) consists of the measurement control system unit. Its function is to carry out the task of measurement and control.

The ground management center node $(\mathrm{Gm})$ is composed of the ground station management system unit, which mainly carries on the task assignment and the control to the ground station.

The relay satellite control node (Rc) is composed of relay satellite control system unit, which mainly carries on the task assignment and the control to the relay satellite ground station

The operational target node( T) consists of objects such as aircraft, missiles, ships, and so on..

$V$ is the set of above nodes.

b)Network edge description of reconnaissance satellite application information chain system

The edge of the information network is used to represent the interaction between nodes. Different 
types of edges can be formed between different nodes. According to the actual situation of combat application, the edge between nodes can be divided into information demand side, task scheduling Edge, control instruction side, remote control edge, information transmission side, service transmission side. $E$ is the set of above edges, i.e. $E=\left\{e_{i j} \mid i, j \in V\right\}$.

c)Network description of reconnaissance satellite application information chain system

On the basis of the above nodes and edges ,the model can be constructed:

$$
\text { RsaicNet }=(V, E, W) \text {; }
$$

Where $V$ is a set of the above nodes and $E$ is a set of the above edges, where $W=\left\{w_{i j}=\left(\right.\right.$ delay $\left.\left._{i j}, p_{i j}\right) \mid i, j \in V\right\}$, delay $i j$ is the the delay time between node $i$ and node $j$, $p_{i j}$ is indicative of the probability of connectivity between node $i$ and node $j$. A simple network model is shown as figure 2 .

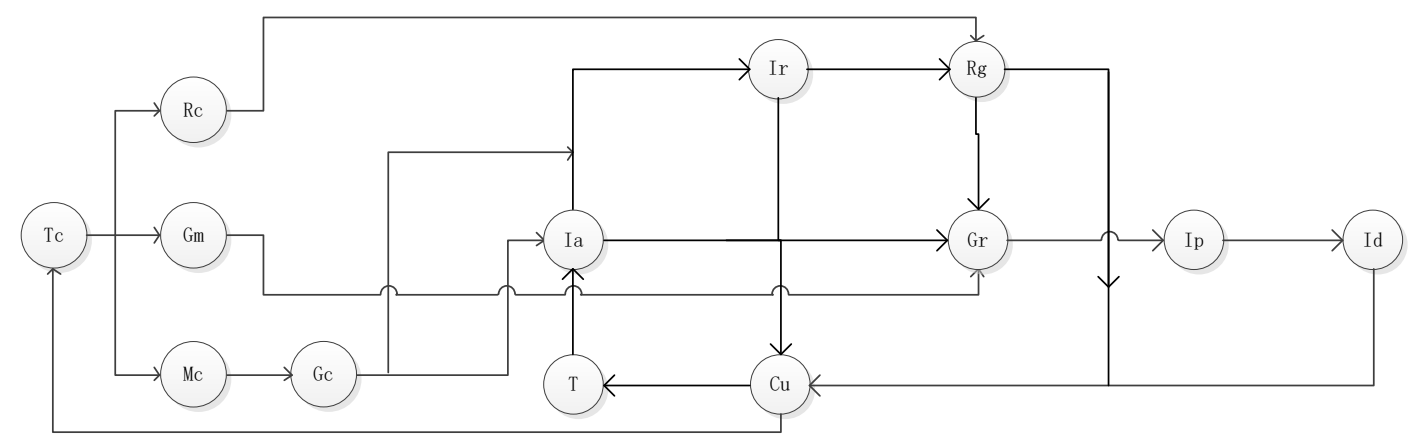

Fig. 2. Network model of reconnaissance satellite information application chain

\section{Network Analysis Method Based on Task Subgraph}

According to the above network model, the performance of the network can be analyzed by using the statistical parameters of complex networks. However, these indicators do not reflect the operational characteristics of reconnaissance satellite application information chain. These measure indicators regard nodes as homogeneous types of nodes, ignoring node heterogeneity, and therefore need a new way to analyze reconnaissance satellite application information chain system network. In this paper, the measurement model of task scheduling subgraph and acquisition application subgraph are put forward based on the network theory and the subgraph theory.

Demand scheduling subgraph is a network subgraph, whose function is to complete the demand scheduling task, as shown in Figure 3.

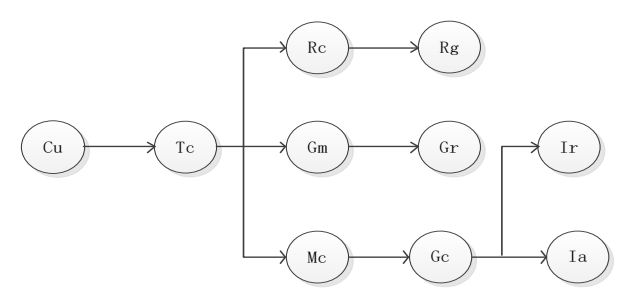

Fig. 2. Demand scheduling subgraph

As can be seen from Figure 3, the demand scheduling sub-graph can be further decomposed into four super-edges. Each super-edge indicates the completion of different task scheduling activities. The four super-sides are described as follows:

Hedge1: $\mathrm{Cu}->$ Tc->Rc->Rg; Hedge2: $\mathrm{Cu}->\mathrm{Tc}->\mathrm{Gm}->\mathrm{Gr}$; 
Hedge3: Cu-> Tc->Mc->Gc->Ir; Hedge4: Cu-> Tc->Mc->Gc->Ia

The completion of the activities of the super-edge can be calculated by the following formula:

$$
\operatorname{delay}(\text { Hedge })=\sum_{e_{i j} \in \text { Hedge }} \text { delay }_{i j}, \overline{p(\text { Hedge })}=\sqrt[n]{\prod_{e_{i j} \in \text { Hedge }} p_{i j}}, \quad n=\mid \text { Hedge } \mid \text {. }
$$

Acquisition application submap is a network subgraph, whose function is to complete the task of information acquisition and information application, as shown in Figure 4.

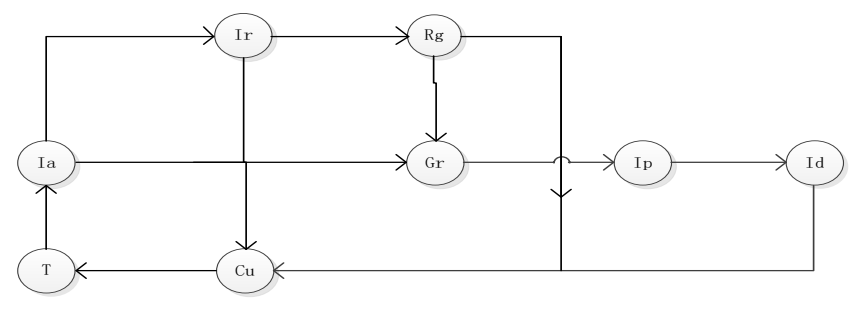

Fig. 3. Acquisition application submap

As can be seen from Figure 4, the acquisition application submap can be further decomposed into five loops. These loops are described as follows:

$$
\begin{aligned}
& \text { Loop1: T->Ia-> Gr->Ip->Id->CU->T; Loop 2: T->Ia->Cu->T; } \\
& \text { Loop 3: T->Ia->Ir->Cu->T; Loop 4: T->Ia->Ir->Rg->Cu->T; } \\
& \text { Loop 5: T->Ia->Ir-> Rg-> Gr->Ip->Id->Cl->T }
\end{aligned}
$$

The completion of the activities of the loop can be calculated by the following formula:

$$
\operatorname{delay}(\text { Loop })=\sum_{e_{i j} \in \text { Loop }} \text { delay }_{i j}, \overline{p(\text { Loop })}=\sqrt[m]{\prod_{e_{i j} \in \text { Loop }} p_{i j}}, m=\mid \text { Loop } \mid \text {. }
$$

Therefore, the network graph of the system can be expressed as the two sub graphs and its time delay and capacity can be determined by the corresponding subgraph.

\section{An Example (omitted)}

\section{References}

[1] C.Y.Wang, H.G.Chen, "Space-based reconnaissance and surveillance system development and military application,” Sichuan Journal of Armament, vol. 11,2011, p. 140-143.

[2] P.L. Jiang, "From space-based strategic reconnaissance to the development of space-based tactical reconnaissance,” Aerospace Electronic Warfare, vol. 6,2014, p. 17-20 +29.

[3] D. G. Qin, L.Y.Chen, "Space-based Information Acquisition System Integrated Command and Control,” Journal of Equipment Academy, vol. 1,2016, pp. 63-68.

[4] G. Liu,L.M.Li,D.S.Qiu,F.F.Tang,"Study on Design Method of Satellite Information Application Chain with Information Center Flow,” Journal of Equipment Academy, vol. 3,2014, pp. 63-66.

[5] W.Du, C.H.Wu, Z.J.Du,et.al. Operational System Network Modeling and Analysis Research Based on C2_Link. Fire Control \& Command Control ,vol. 11,2014,pp.93-96.

[6] K.Luo, M.Z.Zhang, X.Wu. Key nodes analysis model for space information temporal network based on operation loop[J], vol.7,2016, pp.1573-1576. 